\title{
EMBUTIDOS FORTIFICADOS CON PROTEÍNA VEGETAL A BASE DE QUINUA (Chenopodium quinoa Wild.)
}

\author{
Priscila Maldonado ${ }^{1}$
}

\begin{abstract}
RESUMEN
El objetivo de esta investigación es substituir la mayor cantidad de proteína animal, por proteína vegetal a base de quinua, sin disminuir la calidad y aceptabilidad del producto, se optó por este pseudocereal ya que posee características nutricionales muy importantes para la alimentación humana. En la actualidad el país atraviesa por una crisis en la disponibilidad de alimentos y por una dependencia crítica de materia prima importada, por lo cual necesita de alternativas propias, que fomenten el consumo de productos nativos y autóctonos. Al elaborar productos con materia prima disponible en el país logramos disminuir la dependencia externa y promover el trabajo interno y por ende contribuir a garantizar la Soberanía Alimentaria. La Soberanía Alimentaria es el derecho de los pueblos a definir sus propias políticas y estrategias sustentables de producción, distribución y consumo de alimentos que garanticen el derecho a la alimentación para toda la población, con base en la pequeña y medi<ana producción, respetando sus propias culturas y la diversidad de los modos campesinos, pesqueros e indígenas de producción agropecuaria, de comercialización y de gestión de los espacios rurales, en los cuales toda persona desempeña un papel fundamental. La quinua posee un excepcional balance de proteínas, grasa, aceite, almidón y un alto contenido de aminoácidos. Esta investigación se desarrolla en la Universidad Tecnológica Equinoccial, en la cátedra de procesamiento de productos Cárnicos y Pesqueros. Se ha realizado una primera formulación en la cual, se ha sustituido un 30\% de carne animal por quinua, obteniéndose excelentes resultados en cuanto a calidad, composición nutricional y disminución de costos. El valor nutricional se calculó teóricamente y arrojo óptimos resultados.
\end{abstract}

\footnotetext{
${ }^{1}$ Facultad de Ciencias de la Ingeniería, Universidad Tecnológica Equinoccial, Av. Occidental y Mariana de Jesús. Quito, Ecuador. bpriscilamaldonado@hotmail.com
} 


\section{INTRODUCCIÓN}

En un artículo publicado en Febrero del 2009 por Vargas Catering S.A "No hay un cereal más nutritivo e indispensable para el ser humano que la quinua". Es el cereal de mayor y más completa composición en aminoácidos que existe en nuestro planeta. Contiene 20 aminoácidos incluidos los 10 esenciales para el ser humano, especialmente la lisina que es de vital importancia para el desarrollo de las células del cerebro, los procesos de aprendizaje, memorización y raciocinio, así como para el crecimiento físico (Velasco, s.f.).

En el documento técnico "Potencial Nutricional de Harinas de Quinua" realizado por la Facultad de Ciencias Agropecuarias en Colombia, se muestra claramente el contenido de aminoácidos esenciales que contiene la quinua respecto a otras proteínas tanto vegetales como animales (Romo, Rosero, y col, 2007)

Tabla 1. Comparación de Aminoácidos de la Quinua respecto a otras fuentes

\begin{tabular}{|l|c|c|c|c|c|c|c|c|c|c|}
\hline \multirow{2}{*}{ Aminoácido } & Quinua* & Arroz & Maíz & Trigo & Fríjol & Carne & Pescado & Leche & $\begin{array}{c}\text { Patrón } \\
\text { FA0 }\end{array}$ \\
\cline { 2 - 10 } & \multicolumn{8}{|c|}{ gaminoácidos/100 g de proteína } \\
\hline Arginina & 6,8 & 6,9 & 4,2 & 4,5 & 6,2 & 6,4 & 5,1 & 3,7 & 5,0 \\
\hline Fenilalanina & 4,0 & 5 & 4,7 & 4,8 & 5,4 & 4,1 & 37 & 1,4 & 6,0 \\
\hline Histidina & 2,8 & 2,1 & 2,6 & 2 & 3,1 & 3,5 & & 2,7 & 3,0 \\
\hline Isoleucina & 7,1 & 4,1 & 4 & 4,2 & 4,5 & 5,2 & 5,1 & 10 & 4,0 \\
\hline Leucina & 6,8 & 8,2 & 12,5 & 6,8 & 8,1 & 8,2 & 7,5 & 6,5 & 7,0 \\
\hline Lisina & 7,4 & 3,8 & 2,9 & 2,6 & 7 & 8,7 & 8,8 & 7,9 & 5,5 \\
\hline Metionina & 2,2 & 2,2 & 2 & 1,4 & 1,2 & 2,5 & 2,9 & 2,5 & 3,5 \\
\hline Treonina & 4,5 & 3,8 & 3,8 & 2,8 & 3,9 & 4,4 & 4,3 & 4,7 & 4,0 \\
\hline Triftófano & 1,3 & 1,1 & 0,7 & 1,2 & 1,1 & 1,2 & 1 & 1,4 & 1,0 \\
\hline Valina & 3,4 & 6,1 & 5 & 4,4 & 5 & 5,5 & 5 & 7 & 5,0 \\
\hline
\end{tabular}

Fuentes: TAPIA, M y otros; ERPE, INIAP, IICA, GR. $(14,19,20)$

Otro de los beneficios de la quinua es la ausencia total de gluten creando una gran ventaja para las personas celiacas quienes ven una dificultad en el consumo de embutidos por la presencia de harinas con altos contenidos de gluten. 
Tabla 2 Composición Química de granos de Quinua y de Cereales en base seca.

\begin{tabular}{|l|r|r|r|r|r|}
\hline \multicolumn{1}{|c|}{ Elemento } & Quinua* $^{*}$ & Arroz & Cebada & Maíz & Trigo \\
\hline Proteína \% & 16,3 & 7,6 & 10,8 & 10,2 & 14,2 \\
\hline Grasa \% & 4,7 & 2,2 & 1,9 & 4,7 & 2,3 \\
\hline $\begin{array}{l}\text { Carbohidratos } \\
\text { totales \% }\end{array}$ & 76,2 & 80,4 & 80,7 & 81,1 & 78,4 \\
\hline Fibra cruda \% & 4,5 & 6,4 & 4,4 & 2,3 & 2,8 \\
\hline Cenizas \% & 2,8 & 3,4 & 2,2 & 1,7 & 2,2 \\
\hline Energía (kcal/100g) & 399 & 372 & 383 & 408 & 392 \\
\hline
\end{tabular}

Fuentes: TAPIA, METAL, 1979 (29, 30); ERPE, INIAP, IICA, GTZ; 2001 (14)

Desde el año 3000 AC el grano de la quinua (Chenopodium quinoa Wild.) ha sido cultivado en los Andes de Sudamérica. En las áreas montañosas los antiguos Incas, Mayas y Aztecas consideraron la cosecha como un nutriente básico en su alimentación. Según una investigación realizada a las mas importantes empresas de embutidos del país ejecutada por diario Hoy el 25 de Octubre del 2007, determinan que el negocio de los embutidos mueve unos $\$ 120$ millones al año, que el consumo anual en el Ecuador es de 3 kilos por persona y que la demanda crece a una tasa del 5\%.

Si bien no hay cifras exactas sobre el consumo de embutidos a escala nacional, un estudio de Ipsa Group, realizado en Quito y en Guayaquil, determina que, entre las dos ciudades, la primera concentra el 52\%. Sin embargo, los hábitos de consumo en estas urbes son diferentes. En Guayaquil se consume más mortadela (un 37\%) y en Quito, salchichas (63\%).

No es suficiente que el embutido sea nutricionalmente aceptable, este debe cumplir con características de calidad y aceptación como: textura, apariencia, color, sabor etc. 


\section{MATERIALES Y MÉTODOS}

En la elaboración de este embutido se plantearon varias formulaciones, con harina de quinua, con el grano de quinua y con la quinua cocida y deshidratada, obteniéndose una mejor textura con la quinua cocida deshidratada, aparte de mejorar la textura mediante la cocción, mejoramos la digestibilidad y eliminamos los residuos de saponinas presentes en la quinua.

La carne utilizada en esta investigación fue carne de cerdo con $\mathrm{pH}$ de 6,4 y carne de res con $\mathrm{pH}$ de 6,5 que garantizan buena retención de agua ideal para la elaboración de embutidos emulsionados escaldados.

La calidad microbiológica de la carne al inicio del proceso y la higiene durante cada etapa en la elaboración del embutido nos va a garantizar la calidad del producto final.

Dentro de la elaboración del embutido es importante considerar la temperatura en cada etapa del proceso ya que si sobrepasamos las temperaturas de 5 a $10^{\circ} \mathrm{C}$ como máximo la proteína animal se desnaturalizar disminuyendo la capacidad de retención de agua y la capacidad emulsionante.

La formulación base con la cual partimos para proponer varias modificaciones fue una salchicha Suiza. La grasa para la elaboración de embutidos cárnicos emulsionados debe ser dorsal, por tener excelentes características para formar la emulsión, si se utiliza otro tipo de grasa, se generan problemas de ruptura de emulsión o exudación de grasa en el producto final, es importante considerar la frescura de la misma ya que se pueden generar problemas de enranciamiento que afectaran a la calidad organoléptica del embutido.

Tabla 3. Propuesta de formulación con Quinua en grano

\begin{tabular}{|c|c|c|c|c|c|}
\hline & \multicolumn{5}{|c|}{ SALCHICHA TIPO SUIZA } \\
\hline & Formulación Base & 1ra Propuesta & 2da Propuesta & 3ra Propuesta & 4ta Propuesta \\
\hline & \multicolumn{5}{|c|}{$\%$} \\
\hline Carne de cerdo & 30 & 25 & 20 & 20 & 10 \\
\hline Carne de res & 28 & 25 & 20 & 10 & 10 \\
\hline Grasa dorsal de cerdo & 22 & 20 & 20 & 20 & 20 \\
\hline Almidón de papa & 0 & 0 & & & 5 \\
\hline Quinua grano & 0 & 10 & 20 & 30 & 35 \\
\hline Hielo & 20 & 20 & 20 & 20 & 20 \\
\hline Sal & $22 \mathrm{gr} / \mathrm{kilo}$ & $22 \mathrm{gr} / \mathrm{kilo}$ & $22 \mathrm{gr} / \mathrm{kilo}$ & $22 \mathrm{gr} / \mathrm{kilo}$ & $22 \mathrm{gr} / \mathrm{kilo}$ \\
\hline Sales Curantes & $1 \mathrm{gr} / \mathrm{kilo}$ & $1 \mathrm{gr} / \mathrm{kilo}$ & $1 \mathrm{gr} / \mathrm{kilo}$ & $1 \mathrm{gr} / \mathrm{kilo}$ & $1 \mathrm{gr} / \mathrm{kilo}$ \\
\hline Condimentos & $3 \mathrm{gr} / \mathrm{kilo}$ & $3 \mathrm{gr} / \mathrm{kilo}$ & $3 \mathrm{gr} / \mathrm{kilo}$ & $3 \mathrm{gr} / \mathrm{kilo}$ & $3 \mathrm{gr} / \mathrm{kilo}$ \\
\hline
\end{tabular}

Tabla 4. Propuesta de formulación con Harina de Quinua 


\begin{tabular}{|c|c|c|c|c|c|}
\hline & \multicolumn{5}{|c|}{ SALCHICHA TIPO SUIZA } \\
\hline & Formulación Base & 1ra Propuesta & 2da Propuesta & 3ra Propuesta & 4ta Propuesta \\
\hline & \multicolumn{5}{|c|}{$\%$} \\
\hline Carne de cerdo & 30 & 25 & 20 & 20 & 10 \\
\hline Carne de res & 28 & 25 & 20 & 10 & 10 \\
\hline Grasa dorsal de cerdo & 22 & 20 & 20 & 20 & 20 \\
\hline Almidón de papa & 0 & 0 & & & 5 \\
\hline Quinua Harina & 0 & 10 & 20 & 30 & 35 \\
\hline Hielo & 20 & 20 & 20 & 20 & 20 \\
\hline Sal & 22gr/kilo & $22 \mathrm{gr} / \mathrm{kilo}$ & $22 \mathrm{gr} / \mathrm{kilo}$ & 22gr/kilo & $22 \mathrm{gr} / \mathrm{kilo}$ \\
\hline Sales Curantes & $1 \mathrm{gr} / \mathrm{kilo}$ & $1 \mathrm{gr} / \mathrm{kilo}$ & $1 \mathrm{gr} / \mathrm{kilo}$ & $1 \mathrm{gr} / \mathrm{kilo}$ & $1 \mathrm{gr} / \mathrm{kilo}$ \\
\hline Condimentos & $3 \mathrm{gr} / \mathrm{kilo}$ & $3 \mathrm{gr} / \mathrm{kilo}$ & $3 \mathrm{gr} / \mathrm{kilo}$ & $3 \mathrm{gr} / \mathrm{kilo}$ & $3 \mathrm{gr} / \mathrm{kilo}$ \\
\hline
\end{tabular}

Tabla 5. Propuesta de formulación con Quinua Deshidratada

\begin{tabular}{|c|c|c|c|c|c|}
\hline & \multicolumn{5}{|c|}{ SALCHICHA TIPO SUIZA } \\
\hline & Formulación Base & 1ra Propuesta & 2da Propuesta & 3ra Propuesta & 4ta Propuesta \\
\hline & \multicolumn{5}{|c|}{$\%$} \\
\hline Carne de cerdo & 30 & 25 & 20 & 20 & 10 \\
\hline Carne de res & 28 & 25 & 20 & 10 & 10 \\
\hline Grasa dorsal de cerdo & 22 & 20 & 20 & 20 & 20 \\
\hline Almidón de papa & 0 & 0 & & & 5 \\
\hline Quinua cocida Deshidratada & 0 & 10 & 20 & 30 & 35 \\
\hline Hielo & 20 & 20 & 20 & 20 & 20 \\
\hline Sal & $22 \mathrm{gr} / \mathrm{kilo}$ & $22 \mathrm{gr} / \mathrm{kilo}$ & $22 \mathrm{gr} / \mathrm{kilo}$ & $22 \mathrm{gr} / \mathrm{kilo}$ & $22 \mathrm{gr} / \mathrm{kilo}$ \\
\hline Sales Curantes & $1 \mathrm{gr} / \mathrm{kilo}$ & $1 \mathrm{gr} / \mathrm{kilo}$ & $1 \mathrm{gr} / \mathrm{kilo}$ & $1 \mathrm{gr} / \mathrm{kilo}$ & $1 \mathrm{gr} / \mathrm{kilo}$ \\
\hline Condimentos & $3 \mathrm{gr} / \mathrm{kilo}$ & $3 \mathrm{gr} / \mathrm{kilo}$ & $3 \mathrm{gr} / \mathrm{kilo}$ & $3 \mathrm{gr} / \mathrm{kilo}$ & $3 \mathrm{gr} / \mathrm{kilo}$ \\
\hline
\end{tabular}




\section{RESULTADOS Y DISCUSIÓN}

En la primera formulación propuesta que fue a base de quinua en grano, se obtuvo resultados poco favorables, básicamente en la textura del producto ya que se pudo sentir los granos de quinua.

Otra desventaja es que el producto al obtener quinua en grano posee residuos de saponinas y la digestión se hace difícil.

Con la segunda propuesta a base de harina de quinua se generó un producto muy seco y con textura bastante dura, la harina absorbió mucha agua y fue evidente la falta de jugosidad en el producto final.

Con la tercera propuesta a base de quinua cocida y deshidratada se generó la mejor textura en el producto, con esta propuesta se calcularon los siguientes índices.

En la tabla 6 se puede evidenciar el cálculo de los índices de capacidad de retención de agua, capacidad emulsionante, y capacidad de conservación. Estos índices son muy importantes para determinar la calidad del producto final.

El índice de capacidad de retención de agua debe estar en un rango de 4 a 5, para que no exista ruptura de emulsión por exceso de humedad o deficiencia de proteína.

El índice de capacidad emulsionante debe estar en un rango de 1,5 a 2,5, depende del producto que deseamos elaborar, si necesitamos consumir el producto en frío debe poseer menor contenido de grasa, pero si el embutido se va a consumir en caliente puede contener mayor cantidad de grasa.

El índice de conservación debe ser mínimo de 0,04 para que el producto, este relaciona el contenido de humedad con la sal presente en la formulación, en el cuadro se puede evidenciar que a medida que se aumenta la proteína vegetal disminuye el contenido de humedad el mismo que se relaciona directamente con la conservación del embutido.

Tabla 6. Índices sobre Propiedades funcionales

\begin{tabular}{|l|c|c|c|}
\cline { 2 - 4 } \multicolumn{1}{c|}{} & \multicolumn{3}{c|}{ Quinua Deshidratada } \\
\cline { 2 - 4 } \multicolumn{1}{c|}{} & ICRA & ICE & IC \\
\hline 1ra Propuesta & 5,2 & 1,8 & 0,04 \\
\hline 2da Propuesta & 5 & 1,7 & 0,05 \\
\hline 3ra Propuesta & 5 & 1,5 & 0,07 \\
\hline 4ta Propuesta & 4,5 & 1 & 0,09 \\
\hline
\end{tabular}




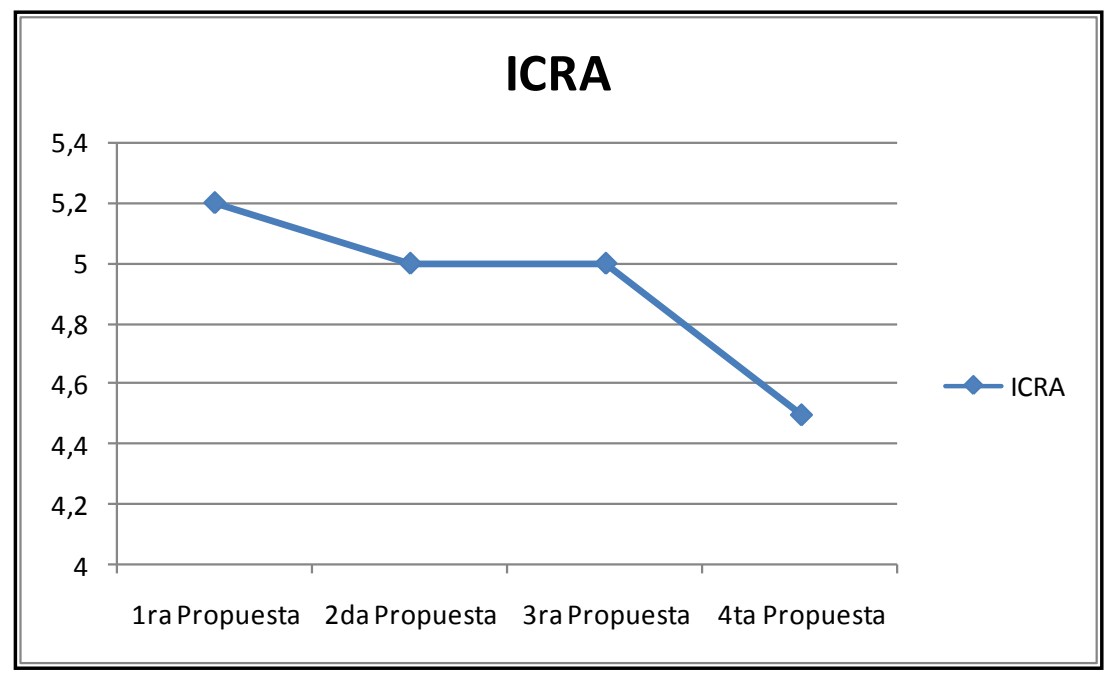

Figura 1. Índice de Capacidad de Retención de Agua

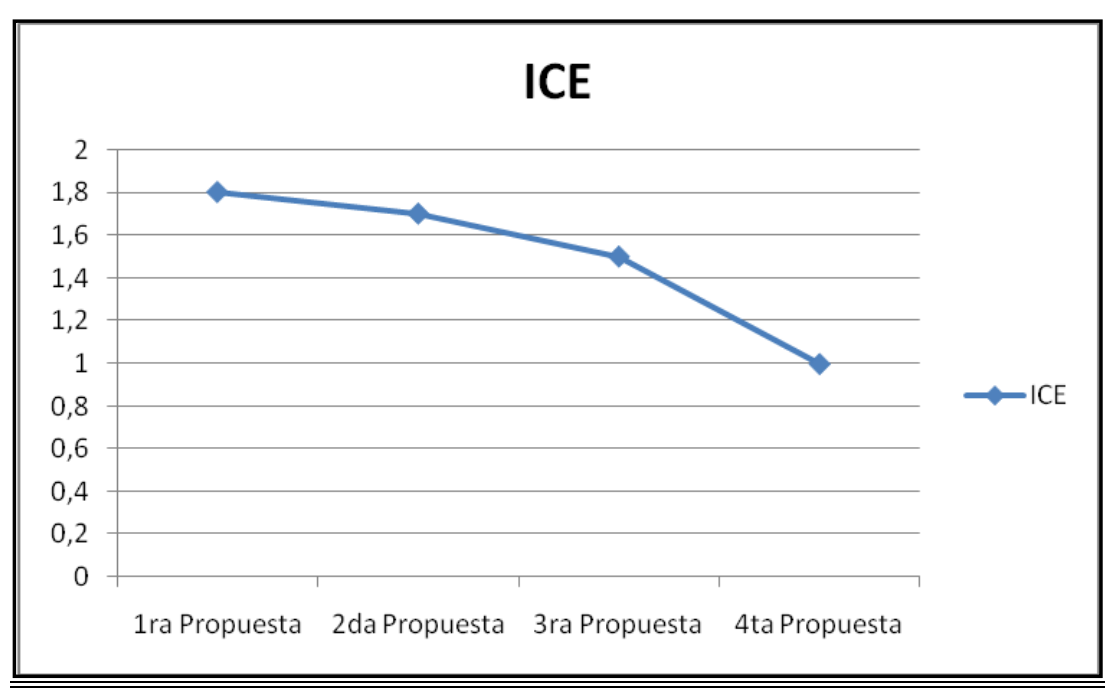

Figura 2. Índice de Capacidad de Emulsión 


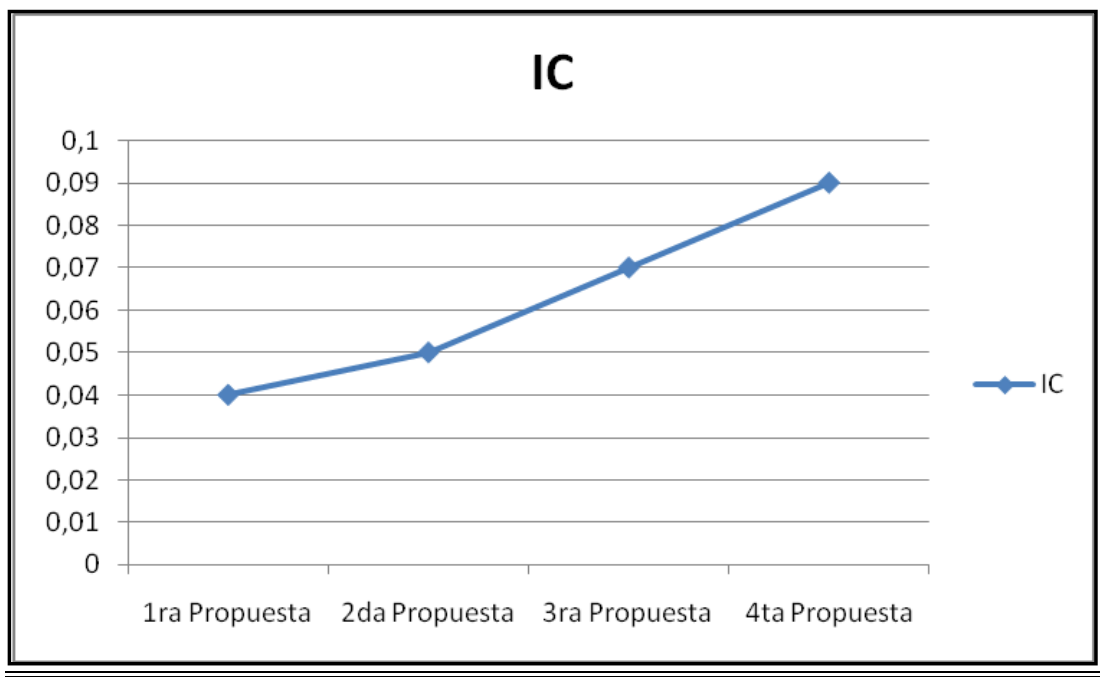

Figura 3. Índice de Capacidad de Conservación

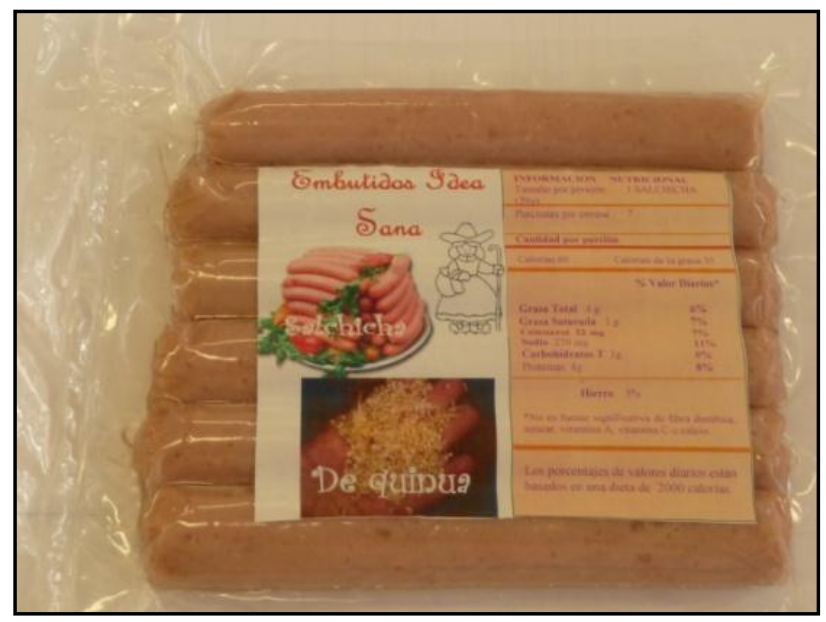

Figura 4. Foto de la Salchicha Suiza con aporte de quinua 


\section{CONCLUSIONES}

En este primer estudio sobre la fortificación del embutido con aporte proteico, se determinó que la mejor formulación es la tercera propuesta, remplazando hasta un 30\% de carne por quinua deshidratada, obteniendo las mejores características organolépticas en el producto final.

Si bien esta investigación debe ser comprobada a nivel de laboratorio con un análisis proximal de la formulación sugerida, el objetivo de esta primera investigación es determinar la factibilidad de remplazar proteína vegetal que encontramos disponible a nivel nacional por proteína animal o proteína vegetal importada como es el caso de texturizados de soya.

El índice de Capacidad de retención de agua se encuentra dentro de parámetros normales lo que nos indica que la emulsión no se romperá.

El índice de emulsión nos recomienda que el producto se pueda consumir en frío ya que no afectará la palatabilidad del mismo.

El índice de conservación nos garantiza que el producto va a durar, pero este índice tiene que ser considerado por factores externos como la calidad de la materia prima y las consideraciones de higiene con las que se procesó. 


\section{BIBLIOGRAFÍA}

Forrest, John. (1975). FUNDAMENTOS DE LA CIENCIA DE LA CARNE. Editorial AcribiaZaragoza. España.

Price, James. (1994). CIENCIA DE LA CARNE Y PRODUCTO CARNICO. Editorial Acribia. 2da. Edición. España.

Wirth, F. (1992). TECNOLOGIA DE EMBUTIDOS ESCALDADO. Editorial ACRIBIA, España 1992.

Velasco, Denise. VALOR NUTRITIVO DELA QUINUA. Artículo Vargas Catering S.A.

Ayala, Guido et al. (2001). Valor nutritivo y usos de la quinua. En: Quinua. Cultivos andinos versión 1.1. FAO. Santiago de Chile.

Bacigalupo, A. y Tapia, M. (1990). Agroindustria. En: Cultivos andinos subexplotados y su aporte a la alimentación. FAO. Santiago de Chile.

Badui, Salvador. (1986). Química de los alimentos. México: Alhambra.

CENTRO DE INVESTIGACIONES, EDUCACIÓN Y DESARROLLO. (2000). Quinua (Chenopodium quinua Willde). Lima. Disponible en Internet: URL: www.agualtiplano.net/cultivos/quinua.htm 